\title{
Dietary perilla oil rich in alpha-linolenic acid prevents systematic inflammation on the endothelium of aorta in apoE knock-out mice
}

\author{
S. H. Hong, M. J. Woo, M. J. Kim and Y. O. Song \\ Department of Food Science and Nutrition and Kimchi Research Institute, Pusan National University, 609-735 Busan, \\ Republic of Korea
}

Systematic inflammation on the endothelium of aorta plays a crucial role in the development and progression of atherosclerosis in individuals with hyperlipidemia. Recently, it has become apparent that decreased bioavailability of endothelial nitric oxide leads to the development of atherosclerosis ${ }^{(1)}$. Tissue specific plasminogen activator at endothelium may retard thrombosis which is found in advanced atherosclerosis $^{(2)}$. Numerous studies have cited that $n-3$ fatty acids have beneficial effects against CVD and its mechanisms studies are also carried out extensively ${ }^{(3)}$.

The aim of this study is to determine the effects of perilla oil (PE), a dietary source for $n-3 \beta$-linolenic acid, on the expression of adhesion molecules, eNOS, and iNOS in the aorta of Apo E knock-out (KO) mice fed with high cholesterol diet. Experimental diet contains $1.25 \%$ cholesterol and $10 \%$ of different oils. Lard (LD), PE and sunflower oil (SN) were used as the source for saturated, $n-3$, and $n-6$ fatty acid, respectively. After 10 weeks of feeding, all the mice were anesthetised with ketamin $(11 \mathrm{mg} / \mathrm{kg}$ b.w.) and the aorta was collected. Adhesion molecules (ICAM-1, VCAM-1), eNOS and iNOS expression on aortic tissues were analysed by immunehistochemical (IHC) staining method using DAB (3,3'-diaminobenzidine) chromogen. Degree of expression for the respective molecule or protein was determined using an Adobe Photoshop CS5 program. Cells that turned into brown color during IHC reaction were counted followed by scanning the area with MIRAX MIDI slide scanner. Fibrinolytic activity was measured by a fibrin plate method using a plasma euglobulin fraction, and data were expressed as plasmin units.

(A) eNOS



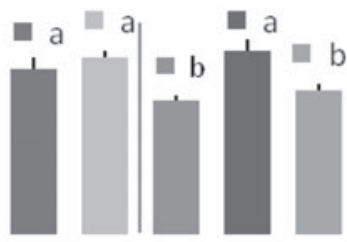

$\overline{\text { Wild type }}$
(B) iNOS
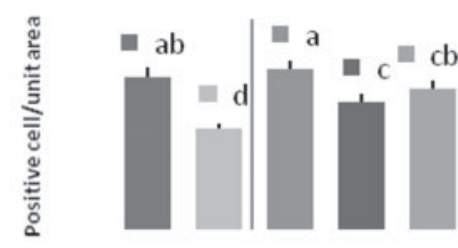

Wild type $\overline{\text { ApoE KO }}$
Table 1. Fibrinolytic activity

\begin{tabular}{lllc}
\hline \multirow{2}{*}{$\begin{array}{l}\text { Mouse } \\
\text { model }\end{array}$} & & \multicolumn{2}{c}{$\begin{array}{c}\text { Lysis area } \\
\text { (plasma units/ml) }\end{array}$} \\
\cline { 3 - 4 } Wild type & Diet & $0.57^{\mathrm{NS}}$ & $\mathrm{SE}$ \\
& $\mathrm{PE}$ & 0.55 & 0.09 \\
& $\mathrm{LD}$ & $0.11^{*}$ & 0.16 \\
apoE KO & PE & $0.22 \dagger$ & 0.03 \\
* & SN & $0.10^{*}$ & 0.03 \\
\multicolumn{2}{c}{ Data with different letters are significantly different with the apoE } \\
KO mice $(P<0.05$, ANOVA, followed by Duncan's test.
\end{tabular}

KO mice $(P<0.05$, ANOVA, followed by Duncan's test.

Fig. 1. Expression of nitric oxide synthase (NOS) in aorta. (A) a, b; (B) a-d Data with

different letters are significantly different with $P<0.05$ (ANOVA, followed by Duncan's test).

ICAM-1 expressions for APE and ASN were decreased compared with the ALD group by 46.59 and $37.84 \%$, respectively $(P<0.05)$. The VCAM-1 for WPE and APE groups were the lowest expression among five experimental groups $(P<0.05)$. The expression of eNOS for the PE group was the highest among ApoE groups, and its activity is comparable with those of wild type mice $(P<0.05)($ Fig. $1 \mathrm{~A})$. iNOS expression for the WPE was lower than that for the WLD group by $33.6 \%(P<0.05)$. In the apoE KO mice, iNOS activity for the PE and SN groups were decreased by 20.8 and $12.5 \%$ than that for the LD group, respectively $(P<0.05)($ Fig. $1 \mathrm{~B})$. As shown in Table 1 , fibrinolysis in apoE KO mice was significantly increased in PE group than LD or SN group $(P<0.05)$. According to these results, PE seems to have vaso-protective effects by elevating the activity of eNOS while suppressing iNOS activity as well as retarding the adhesion molecules expression. Antithrombic effect of PE was also observed. In our previous study, PE demonstrated plasma cholesterol lowering activity in diet-induced hypercholesterolemic ApoE mice. In conclusion, PE might have beneficial effects on atherosclerosis progression through regulating the vascular homeostasis and inhibiting the inflammation in aorta as well as improving of the plasma lipid profiles.

This study was supported by the research grant (no. 109130-3) from the Ministry for Food, Agriculture, Forestry and Fisheries, Republic of Korea, which is gratefully appreciated.

1. Yang Z \& Ming XF (2005) Clin Med Res 4, 53-65.

2. Brunner H, Cockcroft JR, Deanfield J et al. (2005) J Hypertens 23, 233-246.

3. Adkins Y \& Kelley DS (2010) J Nutr Biochem 21, 781-792. 